\title{
Ines Kappstein reicht den Staffelstab weiter
}

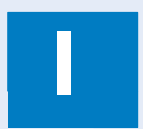

m Frühling 2005 reiste ich für den Georg

Thieme Verlag zu Frau Professor Kappstein nach München. Ich hatte zuvor mit verschiedenen Klinikern gesprochen und erfahren, dass nosokomiale Infektionen unter dem zunehmenden Kostendruck der Krankenhäuser immer ernster genommen wurden. Gleichzeitig herrschte im Spannungsfeld von ÖGD, RKI und verschiedenen „Schulen“ der Krankenhaushygiene große Unsicherheit. Mein Eindruck war, dass es trotz dieser Problematik in Deutschland weder eine wissenschaftliche Fachgesellschaft gab, die nach internationalem Standard evidenzbasierte Leitlinien und Best-Practice-Verfahren veröffentlichte, noch eine eindeutig industrieunabhängige Fach- und Fortbildungszeitschrift, die über den aktuellen, international akzeptierten Stand der Wissenschaft informierte.

Literaturrecherchen und persönliche Empfehlungen führten mich zu Frau Professor Kappstein. Diese war im Gespräch zunächst zurückhaltend. Könnte eine neue Fachzeitschrift zur Prävention nosokomialer Infektionen erfolgreich sein? Doch das up2date-Konzept überzeugte sie schließlich, und je intensiver wir in diesem denkwürdigen Gespräch die Möglichkeiten ausloteten, umso begeisterter zeigte sie sich. Schließlich war sie sogar bereit, die Schriftleiterfunktion zu übernehmen und das, obwohl ihre Tage schon damals randvoll waren mit Aufgaben, die eine weitere anspruchsvolle $\mathrm{Zu}$ satztätigkeit auszuschließen schienen.

Als im Juni 2005 im Verlag die erste Herausgebersitzung mit Frau Professor Kappstein und Herrn PD Dr. Schulze-Röbbecke stattfand, wurde - als erste Amtshandlung - Herr PD Dr. Just telefonisch ins Herausgebergremium berufen. Die Vorbereitungen dauerten dann nochmals etwa ein Jahr, bis die erste Ausgabe der Krankenhaushygiene up2date im September 2006 erscheinen konnte. Seitdem erntet die Zeitschrift sehr viel Lob und Zuspruch - insbesondere von den hygie- nebeauftragten Ärzten, denen die Fortbildungsbeiträge eine große Hilfe sind. Die Zeitschrift, ihre Herausgeber und Autoren belebten dabei die Diskussion im Fachgebiet spürbar: Sie näherten sich alten Zöpfen mit scharfer Schere, indem sie - auf Basis entsprechender Studien - deutlich Stellung bezogen, gelegentlich auch gegen manche „Vorherrschende Meinung“.

In den vergangenen fünf Jahren hat Frau Professor Kappstein mit großem Engagement „den Karren“ der Krankenhaushygiene up2date gezogen - oftmals bei Gegenwind, über Stock und Stein und den Berg hinauf. Sie hat das Profil der Zeitschrift geprägt und oft Übermenschliches geleistet, damit die neue Fachzeitschrift gedeihen möge. Mittlerweile ist die Krankenhaushygiene up2date etabliert, angesehen und wird von allen zur Kenntnis genommen, die auf diesem Gebiet arbeiten oder Verantwortung tragen. Dieser Erfolg ist zu einem ganz großen Teil dem Einsatz, der Begeisterung und dem Engagement von Ines Kappstein zu verdanken. Und so gebührt ihr der herzliche Dank des Verlags, der ihren Entschluss, die Schriftleitung abzugeben, voller Bedauern akzeptieren muss. „Alles hat seine Stunde. Für jedes Geschehen unter dem Himmel gibt es eine bestimmte Zeit.“ (Kohelet 1.3). Frau Professor Kappstein gibt den Staffelstab weiter. Umso mehr freuen wir uns, dass sie der Zeitschrift als Autorin treu bleiben wird.

Gemeinsam mit allen anderen eingebundenen Mitarbeitern des Verlags wünsche ich Frau Professor Kappstein viel Freude beim Ausfüllen der gewonnenen Zeit hin und wieder aber auch ein wenig Langeweile (die sie auf den Gedanken bringen könnte, nochmals einen ihrer ausgezeichneten Beiträge für die Krankenhaushygiene up2date zu verfassen).

\section{Anne C. Repnow für das Team}

„Krankenhaushygiene up2date“

im Thieme Verlag 